\title{
Reversible Photoalignment of Azobenzene in the SURMOF HKUST-1
}

\author{
Tillmann Koehler, Ina Strauss, Alexander Mundstock, Jürgen Caro, and Frank Marlow*
}

Cite This: J. Phys. Chem. Lett. 2021, 12, 8903-8908

Read Online

ABSTRACT: Azobenzene guest molecules in the metal-organic framework structure HKUST-1 show reversible photochemical switching and, in addition, alignment phenomena. Since the host system is isotropic, the orientation of the guest molecules is induced via photo processes by polarized light. The optical properties of the thin films, analyzed by interferometry and UV/vis spectroscopy, reveal the potential of this alignment phenomenon for stable information storage.

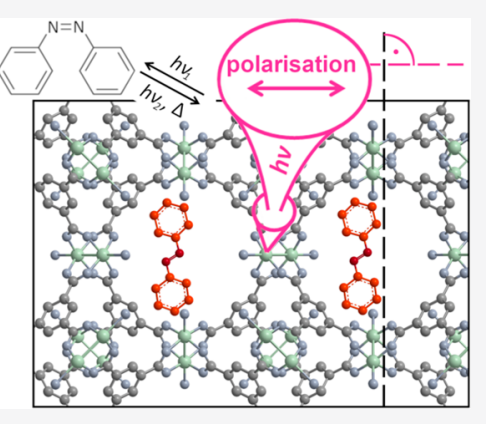

$\mathrm{O}$ ne of the most successful applications of the photochromic molecule azobenzene $(\mathrm{AB})$ and its derivatives is its use as a command layer for liquid crystal displays (LCDs). ${ }^{1-3}$ Here, the molecules are located in a polymer environment and linearly polarized light is used to generate an alignment of the molecule's long axis. This orientation is then transferred to the liquid crystalline layer being forced into a special, e.g., twisted, arrangement in the LCD by the command layers. The photoalignment is rooted in the repeated isomerization of the molecules between the trans $(t-A B)$ and cis $(c-A B)$ states under irradiation which brings the molecular ensemble in a photodynamical equilibrium state. ${ }^{4,5}$ All molecules are continuously switched back and forth until they are, by chance, oriented with their long axis perpendicular to the polarization direction of the light (Figure 1$).^{6}$

The effect of $\mathrm{AB}$ photoisomerization (Figure 1a) has been investigated intensely in solution ${ }^{7}$ and porous environments, e.g., in molecular sieves. ${ }^{8}$ In the latter case, the orientation of the transition moment parallel to the $\mathrm{t}-\mathrm{AB}$ long axis, combined with the constrained anisotropic environment, can be exploited to generate systems with reversibly switchable optical parameters. A large photoswitchable birefringence in AB@ zeolite systems has been reported. ${ }^{9}$ However, alignment of $A B$ molecules can also be achieved by irradiation with linearly polarized light in isotropic environments (Figure $1 \mathrm{~b}$ ). ${ }^{10}$

One drawback of the molecular sieves as porous host for optical applications, despite their promising properties in many regards, is the inability to produce macroscopic crystals or homogeneous thin films. Experiments on zeolite crystals therefore suffer from scattering and low reproducibility of the synthesis. One way to circumvent this limitation is by replacing the host with surface mounted metal-organic frameworks (SURMOFs). For this material class, the ability to produce homogeneous thin films on a substrate has been demonstrated on many different MOF types, ${ }^{11}$ e.g., using layer-by-layer synthesis procedures. ${ }^{12}$ Recent reviews of AB@ MOF systems are available. ${ }^{13,14}$ In pioneering papers, the reversible $\mathrm{AB}$ photoisomerization has been exploited as a molecular valve to close and open the pores of an $\mathrm{AB} @$ SURMOF membrane to switch gas transport. ${ }^{15-17}$ Furthermore, $\mathrm{AB}$ attached as a side group to the linker can be selectively switched using circularly polarized light. ${ }^{18}$ An especially good photoisomerization behavior of $\mathrm{AB}$ was shown in the 3D pores of an HKUST-1 type thin film. ${ }^{19}$ This is possible since two of the framework's three cavity types (with diameters of $1.4 \mathrm{~nm}, 1.1$ and $0.5 \mathrm{~nm}^{20}$ ) are not only spacious enough to accommodate $\mathrm{AB}$ but also provide a free volume bigger than $0.12 \mathrm{~nm}^{3}$, which is needed for photoisomerization of $\mathrm{AB}^{21}$

In the present Letter, we describe a geometrically welldefined AB@HKUST-1 composite system (Figure 1c) with the focus of generating anisotropic optical properties via photoalignment. Since the host crystal is isotropic, we induce an orientation of the guest molecules only via photoalignment, similar to the applications of $\mathrm{AB}$ as a command layer for LCDs. Regarding potential applications, the photoalignment has a big advantage over different photoisomerized states that it does not suffer under the inherent metastability of one of the states and offers easy coupling to birefringence. Furthermore, in this paper, the optical properties of the thin films will be analyzed with angular dependent interferometry $(\mathrm{ADI})^{22}$ with high

Received: July 30, 2021

Accepted: August 27, 2021

Published: September 9, 2021 
a)<smiles>c1ccc(N=Nc2ccccc2)cc1</smiles><smiles>[C]1C2=CC1C2</smiles>

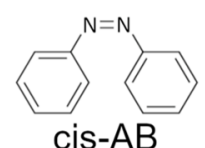

c)

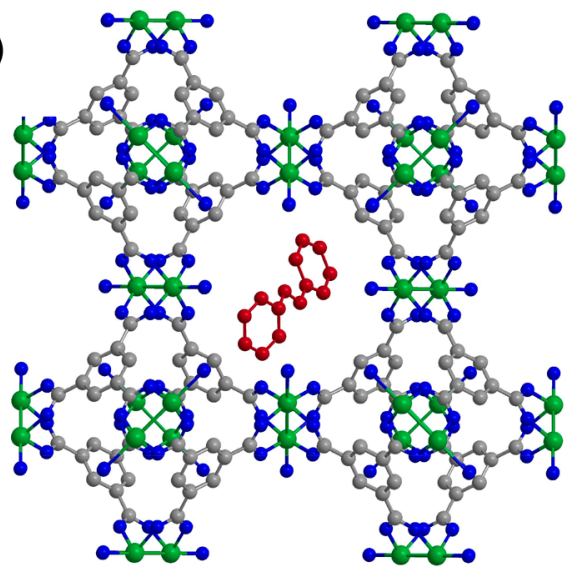

b)

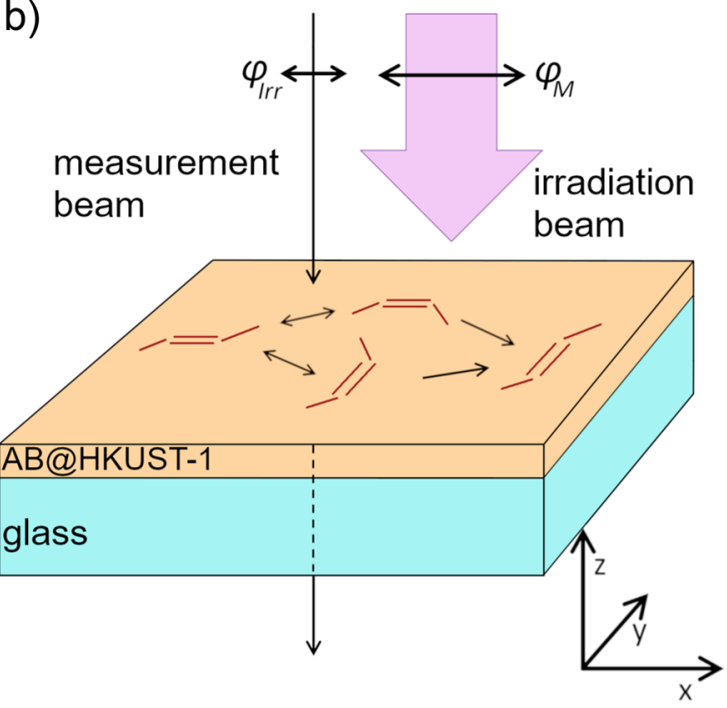

Figure 1. a) Isomerization of $\mathrm{AB}$. b) Experimental scheme and mechanism of photoalignment: The irradiation beam switches the AB; another beam is used to measure absorption. The reorientation is shown on a simplified $A B$ model with the NN double bond and adjacent bonds: $A B$ continuously isomerizes between trans and cis. Excitation of $\mathrm{t}-\mathrm{AB}$ oriented perpendicular to irradiation polarization is unlikely, and therefore, after multiple cycles this orientation is preferentially observed. c) An AB molecule (red) in the HKUST-1 structure with Cu (green), $\mathrm{O}$ (blue), and C (gray) atoms.

accuracy and the stability times of the cis isomer will be determined.

The HKUST-1 MOF-films were prepared layer-by-layer from benzene-1,3,5-tricarboxylic acid and $\mathrm{Cu}(\mathrm{II})$ acetate precursor on FTO glass substrate by a spraying technology, $^{23,24}$ resulting in SURMOFs of optical quality. For $A B$ guest loading, the samples were immersed in an ethanolic solution of $\mathrm{AB}$ for $72 \mathrm{~h}$ at $60{ }^{\circ} \mathrm{C}$. X-ray diffraction showed good agreement with the literature ${ }^{19,25}$ and our previous work on this system (Figure S1). ${ }^{26}$ The nominal loading of the samples was about 1.5 molecules per unit cell, as concluded from UV/vis spectra (see Supporting Information).

The thin films appear slightly colored and expose distinct interference fringes (Fabry-Perot oscillations) in the $\mathrm{UV} /$ vis spectra that can be used to determine the film thickness $d$ and refractive index $n$. In $\mathrm{ADI}^{22}$ this is realized by turning the sample in the spectrometer (for details see the Supporting Information). The interference extrema are blue-shifted with increasing incidence angle $\alpha$ (Figure 2). A multistep process was employed for the data evaluation, as in ref 22 . The thickness of our thin films turned out to be $d=297 \mathrm{~nm} \pm 12$ $\mathrm{nm}$ with a refractive index $n=1.43$. Moreover, the clear occurrence of the Fabry-Perot oscillations shows the excellent SURMOF film quality.

The photoisomerization of the $\mathrm{AB}$ guest was investigated with UV/vis spectroscopy. The sample was irradiated alternately with visible light at $443 \mathrm{~nm}$ and UV light at 365 $\mathrm{nm}$. The most concise way to visualize the isomerization reaction is to plot the change in absorbance $\Delta A$ with reference to a measurement on the same sample (Figure 3 ). Here, the state after the first irradiation with visible light was chosen as reference. A nearly reversible change between two distinct types of spectra, depending on the last irradiation wavelength, was observed.

As known from the literature, ${ }^{4,5}$ the changes can be attributed to an isomerization of the $\mathrm{AB}$ guest molecule by the position of the peaks. The biggest change around $332 \mathrm{~nm}$ is

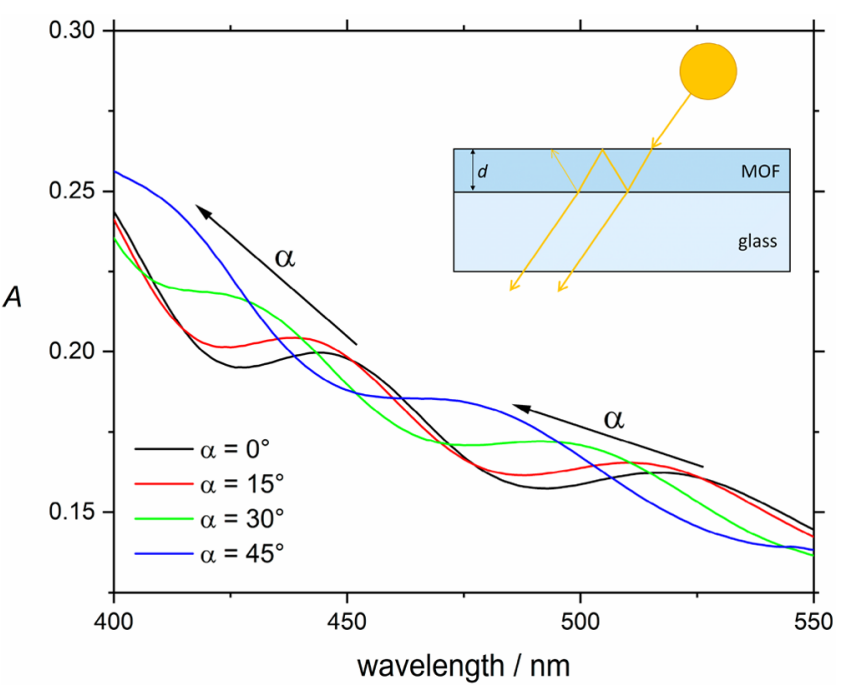

Figure 2. Fabry-Perot oscillations in the absorbance spectrum of the AB@HKUST-1 film. The extrema are blue-shifted with increasing incidence angle $\alpha$ and can be used to determine the optical parameters of the film.

negative and corresponds to a loss of intensity of the $\pi-\pi^{*}$ band of the $\mathrm{t}-\mathrm{AB}$. The $\pi-\pi^{*}$ band of the $\mathrm{c}-\mathrm{AB}$ is expected at much lower wavelengths (and with lower intensities) and is not within the measurement range. The other peak at $434 \mathrm{~nm}$ corresponds to the $n-\pi^{*}$-band, where the $t-A B$ and $c-A B$ positions are found at roughly the same wavelength. The $n-\pi^{*}$ intensity of the $c-A B$, however, is larger than that of the $t-A B$ by a factor of about three and therefore a positive change after irradiation with UV light is expected and was also observed. All positions and intensities depend on the solvent and environment of the $\mathrm{AB}$ molecule and small variations from the literature values can likely be attributed to such effects.

One important parameter in evaluating a photoswitchable system is its stability. Typically, the metastable $\mathrm{c}-\mathrm{AB}$ decays to 


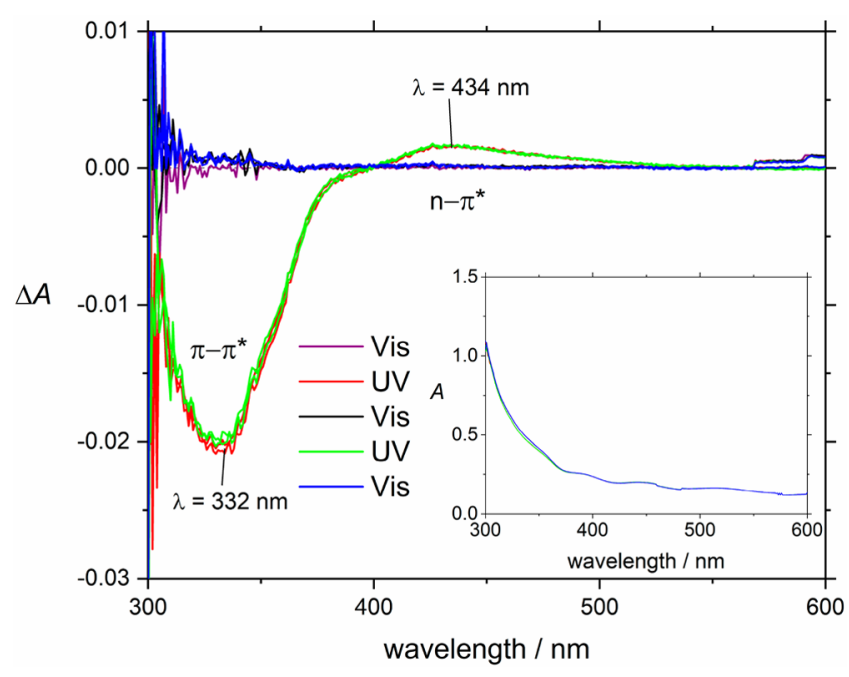

Figure 3. Absorbance change after repeated irradiation with visible and UV light for $3 \mathrm{~min}$. Two spectra were measured after each irradiation, but they varied only little. The dominant $\pi-\pi^{*}$ and weaker $n-\pi^{*}$ bands of the $A B$ are observed at their expected positions. Absolute absorbance spectra are shown in the inset.

$\mathrm{t}-\mathrm{AB}$ in a wide range of time scales, depending heavily on the local environment. We have investigated this process by first irradiating the sample with UV light and then taking UV/vis measurements in regular intervals $\Delta t$. The resulting spectra were evaluated at the minimum of the $\Delta A$ spectrum in the $\pi-\pi^{*}$ band with results shown in Figure 4 . In all cases, the

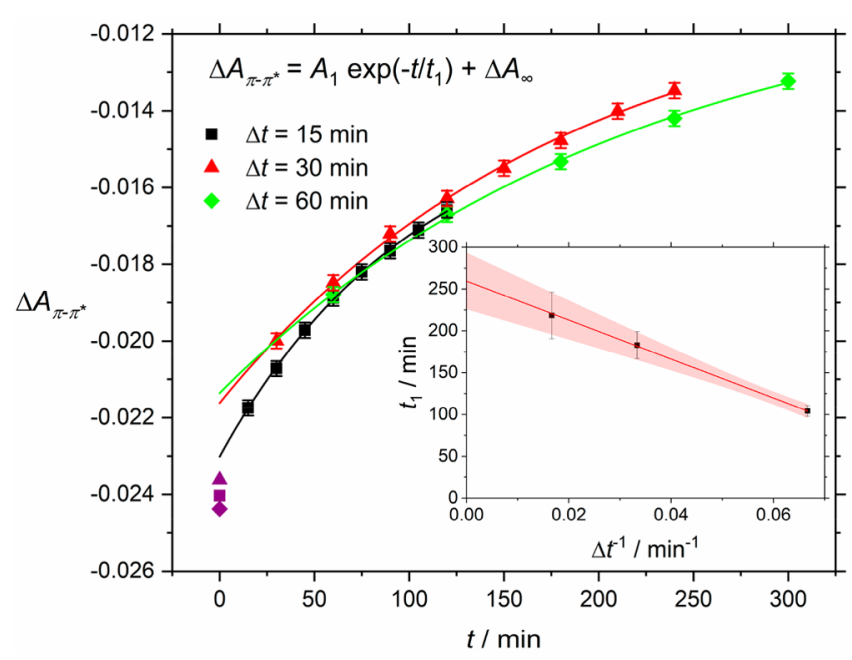

Figure 4. Stability analysis of the cis state. Change in absorbance $\Delta A$ in the $\pi-\pi^{*}$ band plotted against time after irradiation with different $\Delta t$ between the measurement points and exponential fits. Error bars were estimated from the noise of the absorbance data. Inset: Dependence of the decay constants $t_{1}$ vs measurement frequency $\Delta t^{-1}$. A linear fit together with the $95 \%$ confidence area is shown.

trend can be fitted well with an exponential function, but the fit is significantly improved if the first data point is neglected. The decay constant $t_{1}$ of the exponential function shows a linear dependence on measurement frequency $\Delta t^{-1}$, similar as in ref 9. This behavior is caused by the influence of the measurement light. Extrapolating this trend toward zero removes the influence of the measurement and yields $t_{1}=260 \mathrm{~min} \pm 34$ min. This result means $\mathrm{c}-\mathrm{AB}$ relaxes faster in HKUST-1 than it does in solution, where it has a half-life of around 2 days. ${ }^{5,27}$ $\mathrm{AB}$ in molecular sieves shows comparable or a little longer stability times than the values we report here.

During the data evaluation, we excluded the first data points for an improved exponential fit in Figure 4, meaning that the relaxation in the first time period follows a slightly different behavior than during the rest of the measurement. Such effects have been reported before as result of sterically hindered $c-A B$, e.g., in a polymer environment. ${ }^{28}$ We believe that a fraction of the $\mathrm{AB}$ molecules cannot relax completely, because of the interaction with the HKUST-1 matrix.

Following the motivation given above, the switching experiments were finally modified to be sensitive to the orientation of the $\mathrm{AB}$ molecules. To our knowledge, this question has never been investigated in an isotropic porous guest-host system. The sensitivity can be achieved by inserting a linear polarization filter in front of the sample (orientation $\varphi_{M}$ ) and the UV lamp (orientation $\varphi_{I r r}$ ), respectively. Figure 5 shows measurements on AB@HKUST-

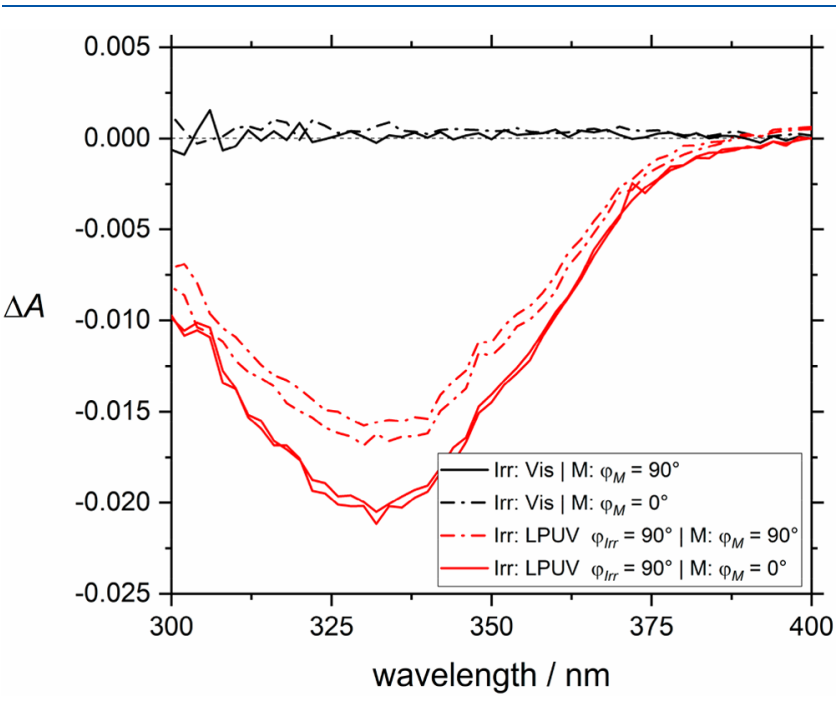

Figure 5. Photoalignment in AB@HKUST-1: absorbance change of the $\pi-\pi^{*}$ band of light polarized vertically (continuous lines) and horizontally (dotted lines). After irradiation with nonpolarized light, no difference between polarizations was observed, while after irradiation with linearly polarized UV (LPUV) light a clear difference can be seen.

1 where after each irradiation two absorption measurements were performed: one polarized horizontally and one vertically. After irradiation with nonpolarized light, the two polarizations show almost no difference, as expected, since the $A B$ is distributed isotropically. After irradiation with linearly polarized UV light, however, higher absorption in the direction perpendicular to the polarization direction of the irradiation light was observed. This effect is consistent with an enhanced orientation of $\mathrm{AB}$ molecules in the perpendicular direction by photoalignment. For additional measurements, see section 2.5 of the Supporting Information.

The influence of the measurement on the aligned state needs to be taken into consideration just as carefully as the influence of the measurement light on the isomer concentrations. In Figure 6 this influence is monitored by two measurements taken in each polarization direction in alternating order. Only a small difference between these two was found. The measurement range and hereby duration of exposure was nevertheless 


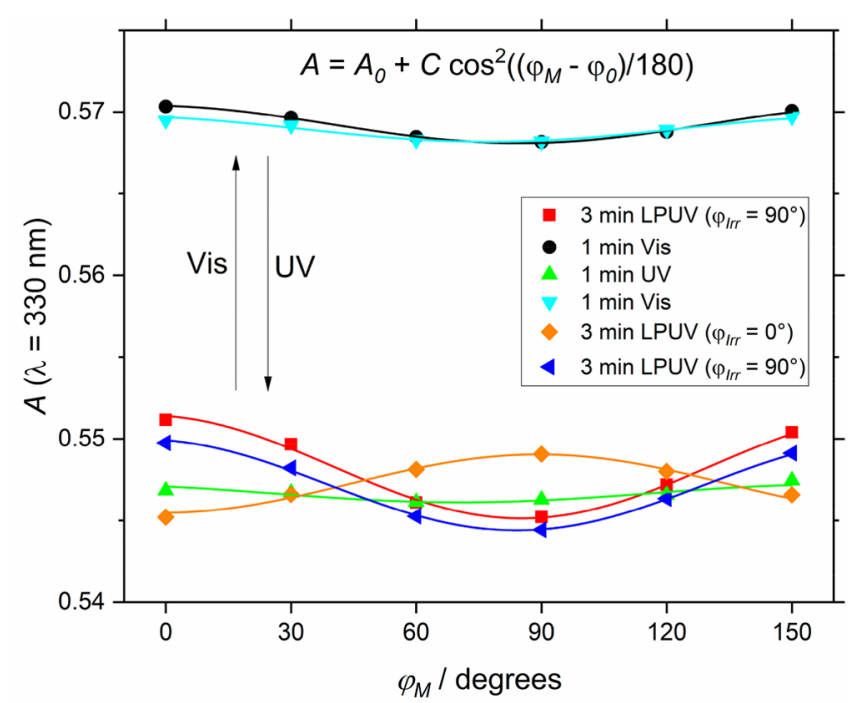

Figure 6. Polarized absorbance at $\lambda=330 \mathrm{~nm}$ after $1 \mathrm{~min}$ of irradiation with the light type indicated in the plot legend. After each irradiation, six data points at different polarization directions $\varphi_{M}$ were collected and fitted with a $\cos ^{2}$ function. The highest absorbance is observed perpendicular to the linearly polarized light $\varphi_{\text {Irr }}$, which is consistent with $\mathrm{AB}$ aligned in this direction.

further reduced to a single spectral data point in the following measurements. Additional angles between the perpendicular and parallel polarization were measured to be able to check the data consistency. The results shown in Figure 6 can be fitted in all cases with a $\cos ^{2}$ function, thus, confirming photoalignment of the $\mathrm{AB}$ guest molecules. ${ }^{29}$

The sequence of measurements is shown by the order in the plot legend in Figure 6, from top to bottom. Considering this, the results reveal two additional surprising characteristics. First, some degree of orientation is preserved even after irradiation with nonpolarized light, both visible and UV. However, every additional irradiation does reduce the degree of orientation. This is seen by the difference of the cyan and the black curve, between which an additional switching step with nonpolarized UV and visible light was performed. Furthermore, an intermediate step of irradiation with visible light was not always needed for the alignment. The state of preferred alignment of the $\mathrm{AB}$ molecule in the vertical direction (orange) was turned around directly toward a preferential orientation in the horizontal (blue).

The strength of the alignment effects can be estimated by comparison with the total photochemical changes. In the data fit formula (see Figure 6), the change in absorption due to the isomerization is described by the difference in $A_{0}$ between the measurements after irradiation with UV to the one with visible light, while the amplitude $C$ of the cosine is a measure of the photoalignment. $A_{0}$ is about four times larger than $C$, suggesting that effectively $1 / 4$ of the $\mathrm{AB}$ molecules are photochemically reoriented inside the pores. This proportion can likely be enhanced by further optimization of the irradiation conditions.

As already noted, photoalignment of $A B$ has been observed before, mostly for $\mathrm{AB}$ derivatives anchored on one end, e.g., to a polymer. ${ }^{29}$ No alignment is expected for $\mathrm{AB}$ in solution, as an oriented state would quickly be lost due to thermal redistribution. The occurrence of photoalignment inside MOF pores, which are sometimes deemed to behave like a "solid solvent", ${ }^{30}$ is therefore by no means trivial. We believe that the guest-host interactions are an important factor in stabilizing the system. This may bypass a general drawback of many photoalignable systems with separated small molecules where the aligned states are not stable. In our system, we have not observed any relaxation process of the alignment degree up to now.

Stability of the cis state for about $4 \mathrm{~h}$ was found. This was plenty enough to perform photoisomerization experiments, but these stability times are too low for applications in data storage. The photoaligned states are, however, not influenced by the same processes. This means information can be much better stored in the orientation of the $\mathrm{t}-\mathrm{AB}$, which is not lost after thermal isomerization. The relaxation of the photoalignment toward an isotropic distribution is described by Brownian motion and the interaction with the environment. ${ }^{29}$

The found refractive index of $n=1.43$ for the SURMOF film requires a closer look. Redel et al. reported the refractive index of HKUST-1 thin films increases from $n=1.39$ to $n=1.55-$ 1.6 by the presence of water inside the pores. ${ }^{31}$ Since our refractive index for the composite $\mathrm{AB} @ H K U S T-1$ is only marginally bigger than in the fully activated state, it is once again indicated that only small amounts of guest molecules are present. This and the small thickness are responsible for the small optical changes observed in this work. These changes are however in the same range as similar systems shown in the literature. ${ }^{14,19}$ Differences can likely be attributed to film thickness, loading procedures, and wavelength of light used for the photoisomerization. The optical effects can certainly be increased with further optimization of these parameters. Some inherent limitations might be foreseen in the loading capacity and in film thickness because of scattering. From the molecular view, loading up to 32 molecules/u.c. seem to be possible if one assumes a dominant adsorption interaction of $\mathrm{AB}$ and the BTC. Optical scattering of the MOF films was not visible up to now.

In addition, we believe that additional strategies for increasing the optical switching effects are needed and photoalignment can be one of them. It can, for example, be followed up by birefringence read-out, where small changes in $\mathrm{AB} @$ host systems have already been used before to induce large transmission changes at specific wavelengths. ${ }^{8,9}$ This would also solve the inherent problem of measurement influence, because read-out wavelengths can be chosen outside of the typical absorption range of $\mathrm{AB}$. We will continue this work by conducting similar experiments on AB@MOF systems.

In this work, the geometry of the switching and measurement light was kept as simple as possible. Both hit the sample parallel to the surface normal and only the polarization direction was varied. It is, however, also possible to irradiate and measure the sample from different angles. In such a case the orientation of the $\mathrm{AB}$ molecules needs to be described by $\mathrm{x}$, $\mathrm{y}$, and $\mathrm{z}$ components. Additional experiments have already shown that the alignment is not limited to the MOF's film plane. It is even possible to align the $\mathrm{AB}$ with nonpolarized light in the direction of the light beam, as was described before in AB@Polymer systems. ${ }^{6}$ 3D anisotropy, instead of the often discussed anisotropy inside a plane, could pave the way for many interesting applications, such as data storage, multicommand switching geometries and neuromorphics.

In summary, photoalignment of $\mathrm{AB}$ in the pores of the HKUST-1 SURMOF was shown to be possible, efficient, and 
stable. The molecules are oriented perpendicular to the polarization direction of switching light. To our knowledge, this is the first time that this has been observed for noncovalently attached azobenzene. Reversible switching between two such aligned states works and more sophisticated switching geometries are possible. Because of the large variety of MOF pore sizes available in the literature, many photoisomerizable molecules can possibly be aligned in a similar way. Moreover, especially enhancement with birefringence switching could lead to a large contrast in the measurable optical effects. Birefringence effects are inherently expected for the photoaligned systems, whereas they are restricted to very special cases when only different isomerization states are used such as in ref 8 .

\section{ASSOCIATED CONTENT}

\section{SI Supporting Information}

The Supporting Information is available free of charge at https://pubs.acs.org/doi/10.1021/acs.jpclett.1c02489.

Experimental details, the XRD measurements, ADI, guest loading calculation, and more details about the cislifetime and photoalignment measurements (PDF)

\section{AUTHOR INFORMATION}

\section{Corresponding Author}

Frank Marlow - Max-Planck-Institut für Kohlenforschung, 45470 Mülheim a. d. Ruhr, Germany; Center for Nanointegration Duisburg-Essen (CENIDE), University of Duisburg-Essen, 47057 Duisburg, Germany; O orcid.org/ 0000-0002-8539-8171; Email: marlow@mpimuelheim.mpg.de

\section{Authors}

Tillmann Koehler - Max-Planck-Institut für Kohlenforschung, 45470 Mülheim a. d. Ruhr, Germany

Ina Strauss - Leibniz Universität Hannover, 30167 Hannover, Germany; 이이.org/0000-0001-8824-4162

Alexander Mundstock - Leibniz Universität Hannover, 30167 Hannover, Germany

Jürgen Caro - Leibniz Universität Hannover, 30167 Hannover, Germany; () orcid.org/0000-0003-0931-085X

Complete contact information is available at: https://pubs.acs.org/10.1021/acs.jpclett.1c02489

\section{Funding}

Open access funded by Max Planck Society.

\section{Notes}

The authors declare no competing financial interest.

\section{ACKNOWLEDGMENTS}

The authors thank W. Schmidt and J. Ternieden for help with XRD measurements and the German Research Foundation for financial support within SPP 1928 COORNETs.

\section{REFERENCES}

(1) Yaroshchuk, O.; Reznikov, Y. Photoalignment of Liquid Crystals: Basics and Current Trends. J. Mater. Chem. 2012, 22, 286-300.

(2) Yu, Y.; Ikeda, T. Alignment Modulation of AzobenzeneContaining Liquid Crystal Systems by Photochemical Reactions. J. Photochem. Photobiol., C 2004, 5, 247-265.
(3) $\mathrm{Yu}, \mathrm{H}$. Recent Advances in Photoresponsive Liquid-Crystalline Polymers Containing Azobenzene Chromophores. J. Mater. Chem. C 2014, 2, 3047-3054.

(4) Knoll, H., Photoisomerism of Azobenzenes. In CRC Handbook of Organic Photochemistry and Photobiology, Horspool, W., Lenci, F., Eds.; CRC Press: 2004.

(5) Rau, H., Photoisomerization of Azobenzenes. In Photochemistry and Photophysics; Rabek, J. D., Ed.; CRC Press: 1989.

(6) Kawatsuki, N. Photoalignment and Photoinduced Molecular Reorientation of Photosensitive Materials. Chem. Lett. 2011, 40, 548554.

(7) Quick, M.; Dobryakov, A. L.; Gerecke, M.; Richter, C.; Berndt, F.; Ioffe, I. N.; Granovsky, A. A.; Mahrwald, R.; Ernsting, N. P.; Kovalenko, S. A. Photoisomerization Dynamics and Pathways of Trans- and Cis-Azobenzene in Solution from Broadband Femtosecond Spectroscopies and Calculations. J. Phys. Chem. B 2014, 118, 8756-71.

(8) Hoffmann, K.; Marlow, F.; Caro, J. Photoinduced Switching in Nanocomposites of Azobenzene and Molecular Sieves. Adv. Mater. 1997, 9, 567-570.

(9) Hoffmann, K.; Resch-Genger, U.; Marlow, F. Photoinduced Switching of Nanocomposites Consisting of Azobenzene and Molecular Sieves: Investigation of the Switching States. Microporous Mesoporous Mater. 2000, 41, 99-106.

(10) Vapaavuori, J.; Bazuin, C. G.; Priimagi, A. Supramolecular Design Principles for Efficient Photoresponsive Polymer-Azobenzene Complexes. J. Mater. Chem. C 2018, 6, 2168-2188.

(11) Haldar, R.; Heinke, L.; Wöll, C. Advanced Photoresponsive Materials Using the Metal-Organic Framework Approach. Adv. Mater. 2020, 32, No. e1905227.

(12) Heinke, L.; Tu, M.; Wannapaiboon, S.; Fischer, R. A.; Wöll, C. Surface-Mounted Metal-Organic Frameworks for Applications in Sensing and Separation. Microporous Mesoporous Mater. 2015, 216, 200-215.

(13) Schwartz, H. A.; Schaniel, D.; Ruschewitz, U. Tracking the Light-Induced Isomerization Processes and the Photostability of Spiropyrans Embedded in the Pores of Crystalline Nanoporous MOFs via IR Spectroscopy. Photochem. Photobiol. Sci. 2020, 19, 1433-1441.

(14) Jiang, Y.; Heinke, L. Photoswitchable Metal-Organic Framework Thin Films: From Spectroscopy to Remote-Controllable Membrane Separation and Switchable Conduction. Langmuir 2021, $37,2-15$.

(15) Müller, K.; Knebel, A.; Zhao, F.; Bleger, D.; Caro, J.; Heinke, L. Switching Thin Films of Azobenzene-Containing Metal-Organic Frameworks with Visible Light. Chem. - Eur. J. 2017, 23, 5434-5438.

(16) Wang, Z.; Knebel, A.; Grosjean, S.; Wagner, D.; Bräse, S.; Wöll, C.; Caro, J.; Heinke, L. Tunable Molecular Separation by Nanoporous Membranes. Nat. Commun. 2016, 7, 13872.

(17) Knebel, A.; Sundermann, L.; Mohmeyer, A.; Strauß, I.; Friebe, S.; Behrens, P.; Caro, J. Azobenzene Guest Molecules as LightSwitchable CO2 Valves in an Ultrathin UiO-67 Membrane. Chem. Mater. 2017, 29, 3111-3117.

(18) Kanj, A. B.; Burck, J.; Vankova, N.; Li, C.; Mutruc, D.; Chandresh, A.; Hecht, S.; Heine, T.; Heinke, L. Chirality Remote Control in Nanoporous Materials by Circularly Polarized Light. J. Am. Chem. Soc. 2021, 143, 7059-7068.

(19) Müller, K.; Wadhwa, J.; Singh Malhi, J.; Schöttner, L.; Welle, A.; Schwartz, H.; Hermann, D.; Ruschewitz, U.; Heinke, L. Photoswitchable Nanoporous Films by Loading Azobenzene in Metal-Organic Frameworks of Type HKUST-1. Chem. Commun. 2017, 53, 8070-8073.

(20) Getzschmann, J.; Senkovska, I.; Wallacher, D.; Tovar, M.; Fairen-Jimenez, D.; Düren, T.; van Baten, J. M.; Krishna, R.; Kaskel, S. Methane Storage Mechanism in the Metal-Organic Framework Cu3(btc)2: An in Situ Neutron Diffraction Study. Microporous Mesoporous Mater. 2010, 136, 50-58.

(21) Oliveira, O. N.; Raposo, M.; Dhanabalan, A. Langmuir-Blodgett and Self-Assembled Polymeric Films. In Handbook of Surfaces and 
Interfaces of Materials; Nalwa, H. S., Ed.; Academic Press: 2001; Vol. 4, pp 1-63.

(22) Konjhodzic, D.; Bretinger, H.; Marlow, F. Structure and Properties of Low-n Mesoporous Silica Films for Optical Applications. Thin Solid Films 2006, 495, 333-337.

(23) Hurrle, S.; Friebe, S.; Wohlgemuth, J.; Wöll, C.; Caro, J.; Heinke, L. Sprayable, Large-Area Metal-Organic Framework Films and Membranes of Varying Thickness. Chem. - Eur. J. 2017, 23, 2294-2298.

(24) Montañez, L. M.; Strauß, I.; Caro, J.; Osten, H. J. Impact of Border Traps in Ultrathin Metal-Organic Framework Cu3(BTC)2 Based Capacitors. Microporous Mesoporous Mater. 2019, 277, 136141.

(25) Fu, W.-Q.; Liu, M.; Gu, Z.-G.; Chen, S.-M.; Zhang, J. Liquid Phase Epitaxial Growth and Optical Properties of Photochromic Guest-Encapsulated MOF Thin Film. Cryst. Growth Des. 2016, 16, 5487-5492.

(26) Yakovenko, A. A.; Reibenspies, J. H.; Bhuvanesh, N.; Zhou, H.C. Generation and Applications of Structure Envelopes for Porous Metal-Organic Frameworks. J. Appl. Crystallogr. 2013, 46, 346-353.

(27) Bandara, H. M.; Burdette, S. C. Photoisomerization in Different Classes of Azobenzene. Chem. Soc. Rev. 2012, 41, 1809-25.

(28) Paik, C. S.; Morawetz, H. Photochemical and Thermal Isomerization of Azoaromatic Residues in the Side Chains and the Backbone of Polymers in Bulk. Macromolecules 1972, 5, 171-177.

(29) Sekkat, Z.; Wood, J.; Knoll, W. Reorientation Mechanism of Azobenzenes within the Trans -> Cis Photoisomerization. J. Phys. Chem. 1995, 99, 17226-1723.

(30) Schwartz, H. A.; Olthof, S.; Schaniel, D.; Meerholz, K.; Ruschewitz, U. Solution-Like Behavior of Photoswitchable Spiropyrans Embedded in Metal-Organic Frameworks. Inorg. Chem. 2017, 56, 13100-13110.

(31) Redel, E.; Wang, Z.; Walheim, S.; Liu, J.; Gliemann, H.; Wöll, C. On the Dielectric and Optical Properties of Surface-Anchored Metal-Organic Frameworks: A Study on Epitaxially Grown Thin Films. Appl. Phys. Lett. 2013, 103, 091903. 